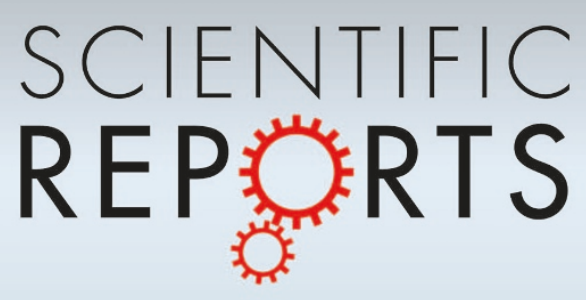

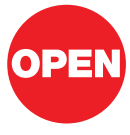

SUBJECT AREAS:

ATOMIC AND MOLECULAR PHYSICS

NANOTECHNOLOGY

NUCLEIC ACID SEQUENCING

SENSORS

Received

18 May 2011

Accepted

11 July 2011

Published

28 July 2011

Correspondence and requests for materials should be addressed to M.T. (taniguti@sanken. osaka-u.ac.jp)

\section{Single-molecule sensing electrode embedded in-plane nanopore}

\author{
Makusu Tsutsui' ${ }^{1}$ Sakon Rahong ${ }^{1}$, Yoko lizumi ${ }^{2}$, Toshiya Okazaki ${ }^{3}$, Masateru Taniguchi' \& Tomoji Kawai \\ ${ }^{1}$ The Institute of Scientific and Industrial Research, Osaka University, 8-1 Mihogaoka, Ibaraki, Osaka 567-0047, Japan, \\ ${ }^{2}$ Department of Chemistry, University of Tsukuba, Tsukuba 305-8577, Japan, ${ }^{3}$ Nanotube Research Center, National Institute of \\ Advanced Industrial Science and Technology, Tsukuba 305-8565, Japan.
}

Electrode-embedded nanopore is considered as a promising device structure for label-free single-molecule sequencing, the principle of which is based on nucleotide identification via transverse electron tunnelling current flowing through a DNA translocating through the pore. Yet, fabrication of a molecular-scale electrode-nanopore detector has been a formidable task that requires atomic-level alignment of a few nanometer sized pore and an electrode gap. Here, we report single-molecule detection using a nucleotide-sized sensing electrode embedded in-plane nanopore. We developed a self-alignment technique to form a nanopore-nanoelectrode solid-state device consisting of a sub-nanometer scale electrode gap in a $15 \mathrm{~nm}$-sized $\mathrm{SiO}_{2}$ pore. We demonstrate single-molecule counting of nucleotide-sized metal-encapsulated fullerenes in a liquid using the electrode-integrated nanopore sensor. We also performed electrical identification of nucleobases in a DNA oligomer, thereby suggesting the potential use of this synthetic electrode-in-nanopore as a platform for electrical DNA sequencing.

A molecular-sized pore in a membrane provides a platform for studying dynamics and structure of polynucleotides in a liquid with single-molecule sensitivity. ${ }^{1-3}$ The sensing mechanism involves detections of ionic current blockade during translocation of an individual molecule through a pore. Considerable efforts have been devoted to apply this nanopore sensor for label-free genome sequencing ${ }^{1-6}$ since the first demonstration of single DNA molecule detection using an $\alpha$-haemolysin channel. ${ }^{7}$ Recent experiments by Clark et al. ${ }^{5}$ adopted an adapter molecule to engineer a protein channel, which contributed to slow down molecular translocation and thereby allowed discriminations of single nucleotides by ionic current. ${ }^{5}$ A graphene nanopore has also attracted much attention because of the well-defined single atomic sheet geometry ideal for DNA sequence detection in conjunction with the excellent mechanical properties. ${ }^{8}$ It remains a challenge, however, to realize single-molecule sequencing by the ionic current blockade method., ${ }^{2,3}$

There is thus growing interest in incorporating additional probes to nanopore sensors. ${ }^{9-11}$ Recent break junction experiments have demonstrated that tunnelling current can serve as a sensitive probe for identifying nucleotides of a single-molecule DNA in liquid environment. ${ }^{12,13}$ Combining this capability, an electrodeembedded nanopore was proposed to be a promising device design for DNA sequencing via transverse electron transport; the device concept is based on electrical identification of nucleotides using the tunnelling current sensing electrodes while a DNA translocates through the pore..$^{2,3,14}$

Despite the huge potential, construction of DNA-sized electrode-embedded nanopore structures has been hindered by the technical difficulty of adjusting positions of a pore and an electrode gap with sub-nanometer precision. $^{15,16}$ Therefore, we developed a self-alignment technique to form a molecular-scale nanoelectrodenanopore system. Our approach is based on electromigration-induced breaking of a $\mathrm{SiO}_{2} / \mathrm{Au} / \mathrm{SiO} \mathrm{O}_{2}$ multilayer structure defined on a silicon wafer (Fig. 1a). As current flows only through $\mathrm{Au}$, the $\mathrm{SiO}_{2}$ layers are expected to remain intact during the electrical breakdown. In this way, virtually perfect alignment of an electrode gap and a pore can be achieved.

\section{Results}

Electrode-embedded nanopore structure was formed by first electrically thinning a $15 \mathrm{~nm}$ thick Au nanojunction sandwiched between $\mathrm{SiO}_{2}$ layers in a vacuum by applying bias sweeps cyclically under an automated resistance feed-back control to induce electromigration at the narrowest constriction until the resistance became larger than $1000 \Omega$ (see Supplementary Fig. S1 and S2). Subsequently, we let the contact break spontaneously at $V_{\mathrm{b}}=0.1 \mathrm{~V}$ and formed a sub-nanometer electrode gap (Fig. $1 \mathrm{~b}$ and $1 \mathrm{c}$, see also Methods). ${ }^{17,18}$ Scanning electron microscope was implemented at various electron beam acceleration voltage conditions $E_{\text {acc }}$ to characterize the 
a

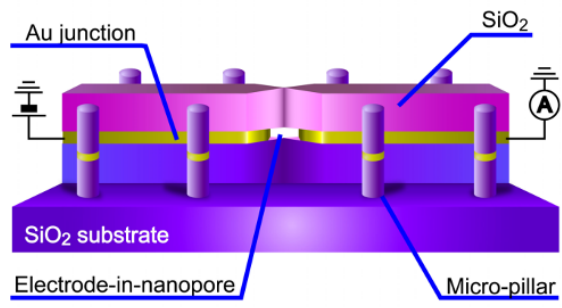

c

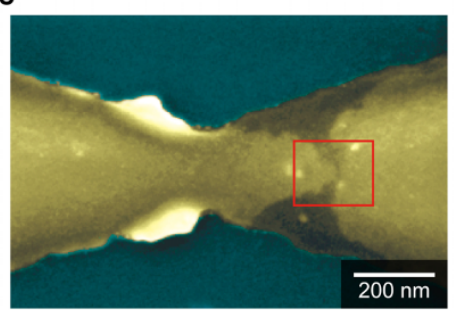

e

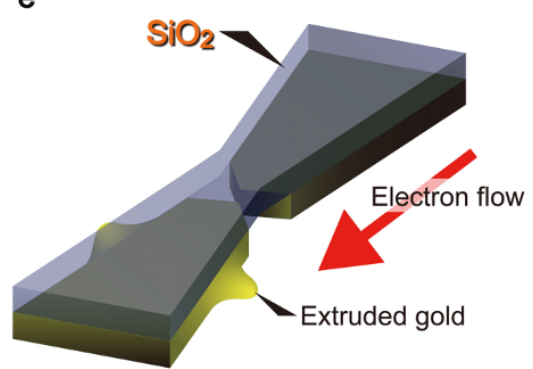

b

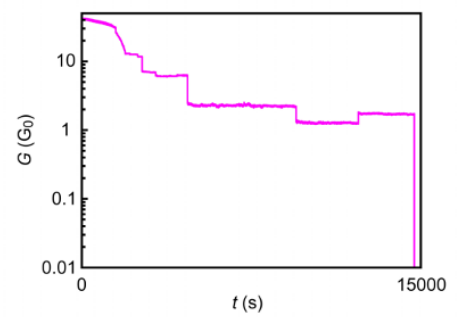

d

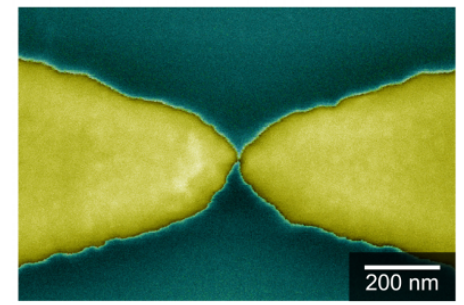

f

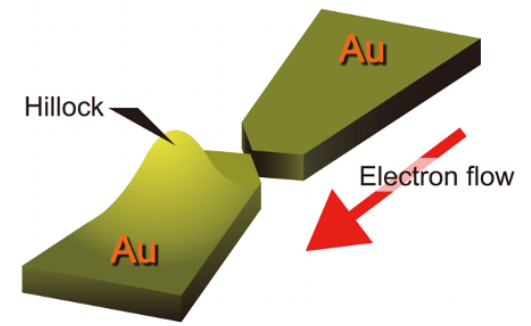

Figure 1 Fabrication of electrode-embedded in-plane nanopore structure. a, Schematic description of a microstructure used to form molecular-sized electrode-embedded nanopore consisting of a $\mathrm{SiO}_{2} / \mathrm{Au} / \mathrm{SiO}_{2}$ multi-layer junction and micro-pillars on a $\mathrm{SiO}_{2} / \mathrm{Si}$ wafer. A gap is formed at the narrowed constriction of the Au layer by electromigration/self-breaking technique. In this way, the electrode gap and the nanoscale pore are automatically self-aligned to each other. b, Conductance versus time $(G-t)$ trace during electrical breakdown of the multi-layer junction. The Au layer was first thinned gradually by current-induced electromigration (see Supplementary Fig. S2). The resulting atom-sized contact was then fractured spontaneously under a low constant voltage of $0.1 \mathrm{~V}$. This self-breaking technique allowed formation of a sub-nanometer scale electrode gap. c-d, False-color scanning electron microscopy images of electrode gaps formed by the electromigration/self-breaking technique for $\mathrm{SiO}_{2} / \mathrm{Au} / \mathrm{SiO}{ }_{2}$ (c) and Au junction (d). The observations were conducted at the electron beam acceleration voltage of $15.0 \mathrm{kV}$ and $5.0 \mathrm{kV}$ for (c) and (d), respectively. Red square points to the location where electrode-embedded nanopore structure has been formed (see Supplementary Fig. S3 and S4). e-f, Electrode gap formation mechanism for the multi-layer junction (e) and the Au contact (f). Atom migration occurs in three dimension when there is no overlayer, leading to growth of a hillock (d,f). In contrast, $\mathrm{SiO}_{2}$ top layer forbids atomic flux in the cross-plane direction, forcing atoms to extrude at the side surface of the Au layer (c,e).

$\mathrm{SiO}_{2}$ cover layer after the electrical junction breaking (see Supplementary Fig. S3 and S4). A flat topology instead of an electrode gap was imaged at $E_{\mathrm{acc}}=0.5 \mathrm{kV},{ }^{19}$ thereby validating that $\mathrm{SiO}_{2}$ overlayer indeed remained undamaged and that the nanopore-withtransverse-electrode structure consisting of a sub-nanometer electrode gap with a $15 \mathrm{~nm}$ sized pore has been formed.

The nanogap-nanopore formation mechanism can be inferred from the electron micrographs. In the active electromigration breaking processes, concentrated Joule heating at the high-resistance constriction increases the mobility of $\mathrm{Au}$ atoms there and induces an atomic flux along a direction of electron flow by wind force. ${ }^{20}$ In case when the $\mathrm{SiO}_{2}$ top layer is absent, migration occurs in three dimension yielding a hillock on the current downstream side of electrode gaps (Fig. 1d and 1f). Situation is different for $\mathrm{SiO}_{2} / \mathrm{Au} / \mathrm{SiO}_{2}$ junctions. It is known that the weak binding energy of $0.1 \mathrm{eV}$ for Au with $\mathrm{SiO}_{2}$ and the large atomic size of $\mathrm{Au}$ give rise to a high barrier that impedes $\mathrm{Au}$ atoms for migrating vertically into $\mathrm{SiO}_{2}$. The critical temperature for $\mathrm{Au}-\mathrm{SiO}_{2}$ migration is roughly defined by the eutectic point of the Au-Si system, which is about $640 \mathrm{~K} .{ }^{21}$ Since the junction temperature cannot be this high during the electromigration processes (although somewhat dependent on the geometry of junctions, electromigration is usually triggered at a temperature around $400 \mathrm{~K}$ to $450 \mathrm{~K}),{ }^{20,22}$ the vertical migration of $\mathrm{Au}$ atoms is unlikely to take place. The $\mathrm{SiO}_{2}$ layer thus effectively impedes Au atomic migration in a cross-plane direction. Consequently, electromigration thinning proceeds via extrusion at side surface of Au contact (Fig. 1c and 1e).

Similarly, it is worth noting that migration of Cr into a Au thin film occurs at a relatively low temperature around $400 \mathrm{~K} .^{23}$ Joule heating during electromigration breakdown process may thus induce vertical migration of thermally exited $\mathrm{Cr}$ atoms into $\mathrm{Au}$, which would lead to a change in impedance of the junction and concomitant error in the feed-back control of the electrical junction breaking. Nevertheless, $\mathrm{Cr}$ layer is only 1 to $2 \mathrm{~nm}$ thick, very thin compared to the thickness of $\mathrm{Au}$ junctions (15 nm thick). Hence, it is anticipated that the alloying effects of the small amount of $\mathrm{Cr}$ in $\mathrm{Au}$ on the electrical properties of the junction poses only marginal influence on the nanopore formation process.

Having established a self-alignment method to fabricate molecularsized electrode-embedded in-plane nanopore structure, we assessed the device potential as a single-molecule detector by conducting electrical detection of fullerenes in a liquid environment. For this purpose, we prepared a fluidic channel of PDMS on the substrate (Fig. $2 \mathrm{a}$ and 
2b; see also Methods and Supplementary Fig. S5). Micro-pillars were also built around the multi-layer junction that served as spacer for the PDMS (Fig. 2c and 2d). Here, we employed Er@C82 as a target. This molecule is chosen to mimic a transverse tunnelling current detection of DNA; diameter of Er@C82 is about $0.8 \mathrm{~nm}$ close to that of nucleotides. ${ }^{24}$

After forming an electrode-nanopore system by the aforementioned self-alignment scheme, we injected a dilute chlorobenzene solution of Er@C82 $(1 \mu \mathrm{M})$ into the fluidic channel and carried out current measurements at $V_{\mathrm{b}}=0.1 \mathrm{~V}$ using the sensing electrodes. As a result, we observed a jump of the current $I$ that indicates trapping of single metalencapsulated $\mathrm{C}_{82}$ molecule by a strong fullerene-Au van der Waals interaction with a binding energy of about $1 \mathrm{eV}$ (Fig. 2e inset). ${ }^{25,26}$ Following the molecular trap, current tended to fluctuate substantially until dropping to zero (Fig. 2e). The I-fluctuation is attributable to Brownian motion of the fullerene molecule tightly bound to $\mathrm{Au}$ electrodes, whereas the current drop to zero indicates molecular detrapping from the electrode gap. A histogram constructed with the $I$ - $t$ curve revealed a pronounced peak at $I_{1} \sim 35 \mathrm{nA}$ and broad peaks at $I_{2} \sim 66 \mathrm{nA}$ and $I_{3} \sim 116 \mathrm{nA}$ (Fig. $2 \mathrm{f}$ ). These peaks are naturally ascribed to electron transport through one, two, and three fullerene molecules anchored to Au electrodes in parallel from the fact that approximately $I_{i}=35 \times i \mathrm{nA}(i=1,2,3 \cdots)$. ${ }^{27}$ Relatively narrow distributions of $I$ reflects the spherical morphology of Er@C82 that provides conformation-independent electronic coupling to Au electrodes. ${ }^{28}$ This well-defined single molecule conductance enabled the electrical single-molecule counting of Er-encapsulated $\mathrm{C}_{82}$ in the organic solvent.

The above results ensure that the size of an electrode gap in the in-plane nanopore is about $0.8 \mathrm{~nm}$, small enough for detecting single-nucleotides by tunnelling current. We therefore utilized the nanoelectrode-nanopore structure for electrical identification of a

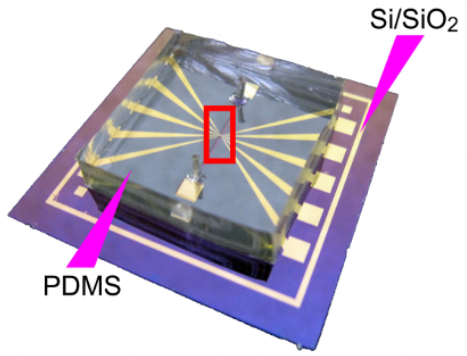

C

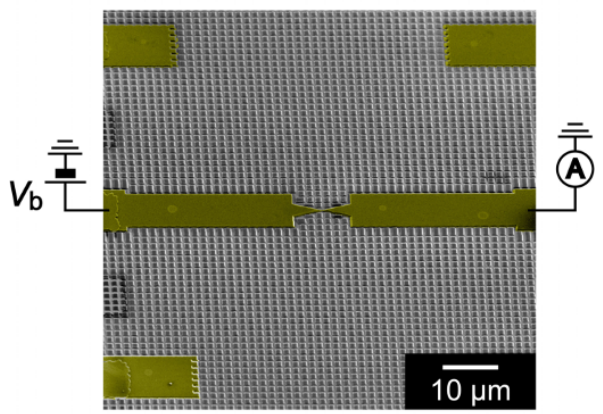

e

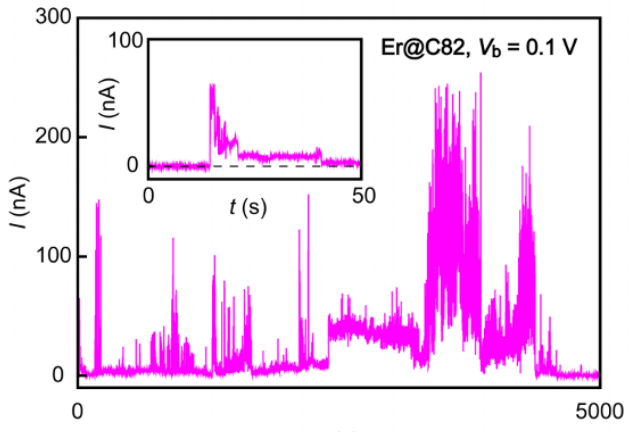

b

Fluid channel (Width $\sim 0.4 \mathrm{~mm}$ )

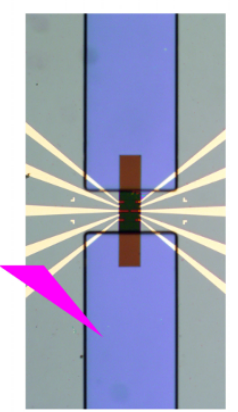

d

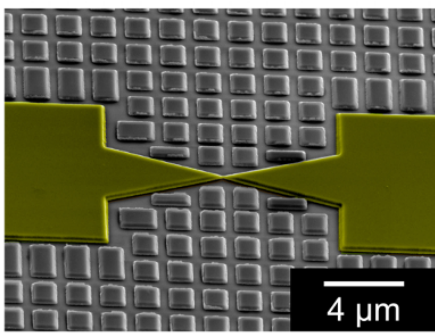

f

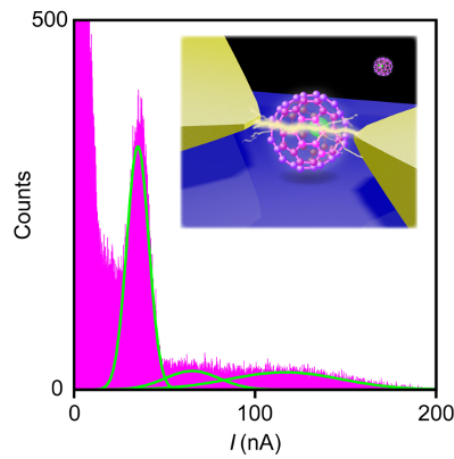

Figure 2 Single-molecule counting using nanopore sensing device. a, A stereoscope image of the in-plane nanopore device. PDMS channel was bonded onto the $\mathrm{SiO}_{2} / \mathrm{Si}$ substrate. b, Optical microscope picture showing the main structure of the nanopore sensor. The fluidic channel was used to flow a solution of a target molecule into the nanoelectrode/nanopore. $\mathrm{c}-\mathrm{d}$, Scanning electron micrographs of the main structure (c) and a magnified view of the $\mathrm{SiO}_{2} / \mathrm{Au} / \mathrm{SiO}_{2}$ multi-layer junction (d). The micro-pillar array was used as a spacer for the PDMS channel (see Supplementary Fig. S5). e, $I-t$ trace measured in a dilute chlorobenzene solution of Er@C82 molecules at the bias voltage of $V_{\mathrm{b}}=0.1 \mathrm{~V}$. A sudden jump of $I$ was observed, indicative of single molecule trapping in the electrode gap (inset). Subsequently, the two-probe current demonstrated fluctuation and dropped to zero after about 5000 seconds signifying molecular detrapping. f, Current histogram revealing a multi-peak profile. Green lines are the Gaussian fit to the $I$ distribution. These peaks are located approximately at $I_{i}=35 \times i \mathrm{nA}(i=1,2,3 \cdots)$. 
nucleotides in a DNA oligomer (Fig. 3a and 3b). Figure $3 c$ displays a $I$ - $t$ trace measured at $V_{\mathrm{b}}=0.75 \mathrm{~V}$ in a Milli-Q solution of guanosine5 '-monophosphates (GMP). We detected spike-like signals representing single-GMP trapping/detrapping events in the electrode gap (Fig. 3d). ${ }^{12,29} \mathrm{~A}$ histogram is constructed by extracting the height of current spikes $I_{\mathrm{p}}$ for 1000 signals (Fig. 3e). The characteristic single-molecule conductance $G_{\mathrm{GMP}}$ deduced from a peak position of the $\log _{10}\left(I_{\mathrm{p}}\right)$ distribution is $G_{\mathrm{GMP}} \sim 111 \mathrm{pS}$, which is in good accordance with $G_{\mathrm{GMP}} \sim 130 \mathrm{pS}$ reported in previous break junction experiments. ${ }^{12}$

The single-molecule analysis was extended to DNA oligomers. In case of 6-mer oligonucleotides (TTTGGG, $1 \mu \mathrm{M}$ in Milli-Q,
$\left.V_{\mathrm{b}}=0.75 \mathrm{~V}\right), I-t$ traces frequently demonstrated two-level steps (Fig. 3f). Correspondingly, $I_{\mathrm{p}}$ histogram manifests a bimodal distribution (Fig. 3g). These peaks are located at $I_{\text {low }} \sim 40$ pA (53 pS) and $I_{\text {high }} \sim 79 \mathrm{pA}(105 \mathrm{pS})$. The latter profile corresponds to that of GMP. Thus, $I_{\text {low }}$ and $I_{\text {high }}$ can be assigned to the conductance states of thymine and guanine in the oligomer, respectively. ${ }^{12}$ It is noticeable that the thymine peak is much smaller than that of guanine in spite of the fact that the oligonucleotides possess equal amount of $\mathrm{T}$ and $\mathrm{G}$ in the sequence. This discrepancy stems from the definition of $I_{\mathrm{p}}$ that tends to overestimate the counts of high-conductance nucleotides in DNA oligomers; $I_{\mathrm{p}}$ is prone to be that of the nucleotides with higher conductivity for the case of multi-level signals. Nonetheless, in this a

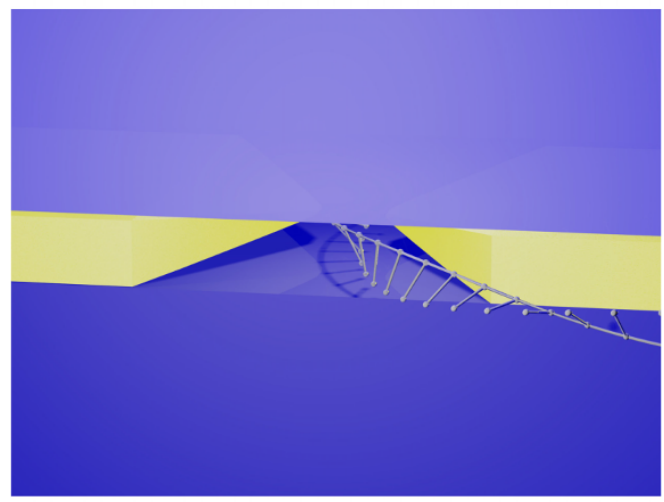

b

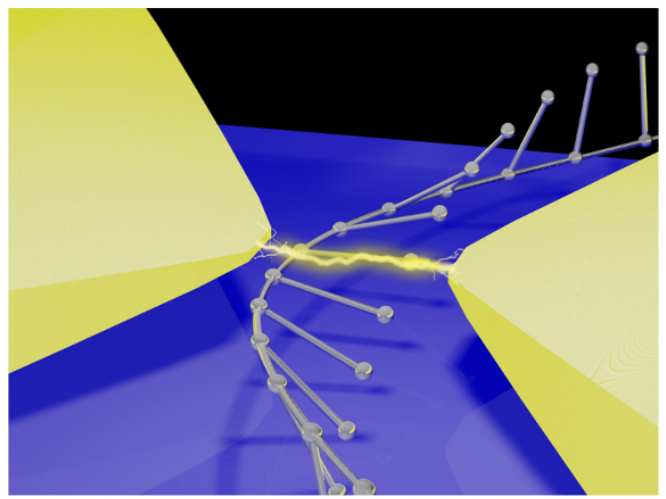

C

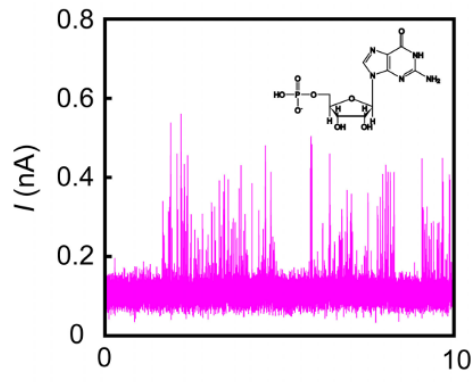

$t(\mathrm{~s})$ d

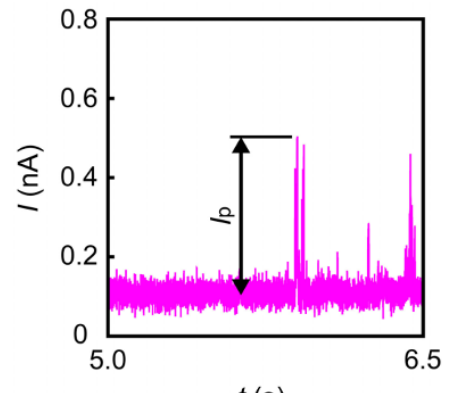

$t(\mathrm{~s})$

e

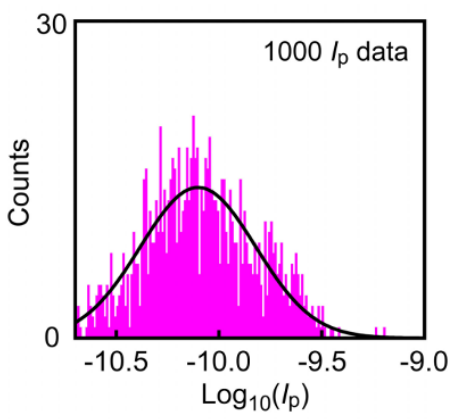

g

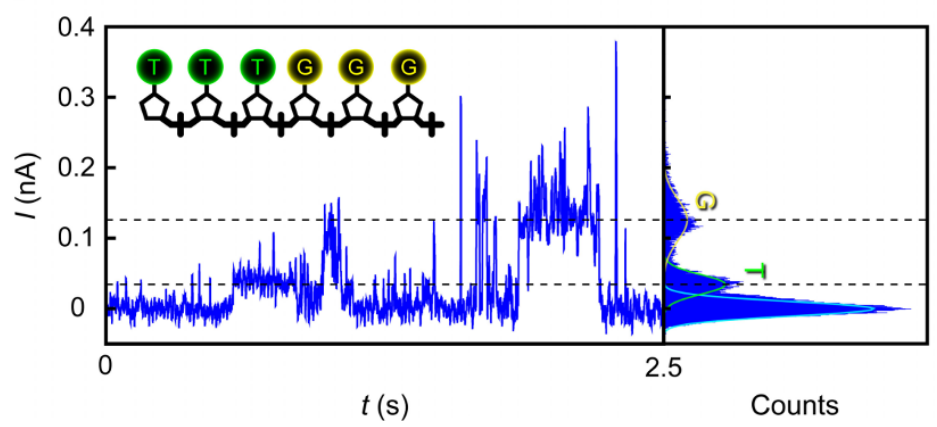

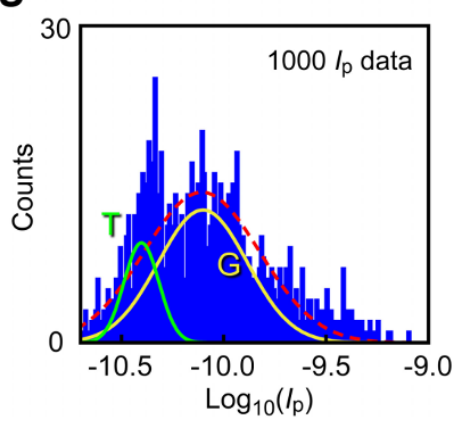

Figure 3 Electrical identification of nucleotides in a single DNA oligomer. a-b, Schematic view of the electrode-embedded in-plane nanopore detector (a) and single-nucleotide identification by tunnelling current (b). c, I-t curve acquired in a Milli-Q solution of guanosine $5^{\prime}$-monophosphate (GMP) at $V_{\mathrm{b}}=0.75 \mathrm{~V}$. The molecular structure of GMP is displayed in the inset. Current spikes were detected signifying trapping/detrapping of single GMP molecule between the pair of Au nanoelectrodes. d, A close view of $I$-spikes. The amplitude of the spike signals $I_{\mathrm{p}}$ is defined as the maximum current attained in a single spike subtracted by the average base current. e, $\log _{10}\left(I_{\mathrm{p}}\right)$ histogram of GMP constructed with $1000 I_{\mathrm{p}}$ data. Black line is a Gaussian fitting. f, $I-t$ trace of 6-mer oligonucleotides with TTTGGG sequence (left) and corresponding current histogram (right). The solid lines are a Gaussian fit to the current distribution. The dotted lines are a guide to the eyes for the peak positions in the histogram. g, $\log _{10}\left(I_{\mathrm{p}}\right)$ histogram of TTTGGG oligomer built with 1000 spike signals. Green and yellow solid lines are a Gaussian fitting to the histogram. Red dashed line is the $I_{\mathrm{p}}$ distribution of GMP. The relatively small peak assigned to thymine is due to the definition of $I_{\mathrm{p}}$ that tend to overestimate the counts of high-conductance nucleotides. 
a

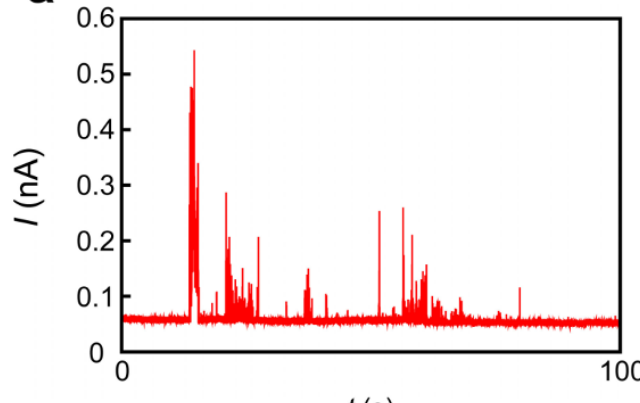

$t(\mathrm{~s})$

C

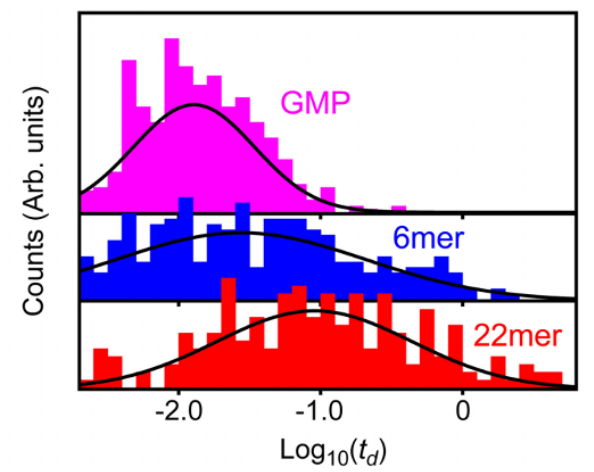

b

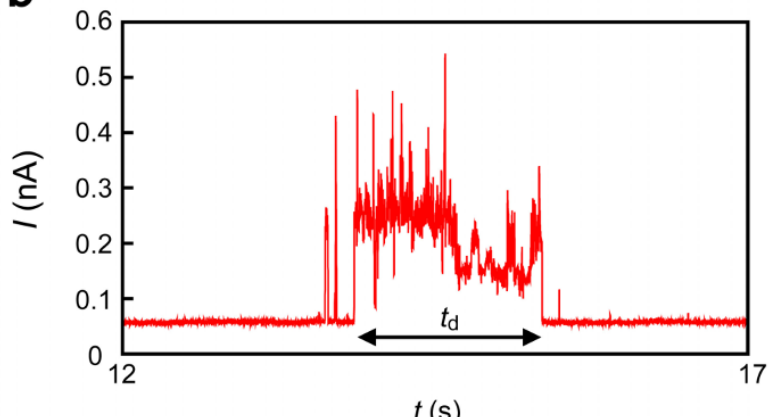

$t(\mathrm{~s})$

d

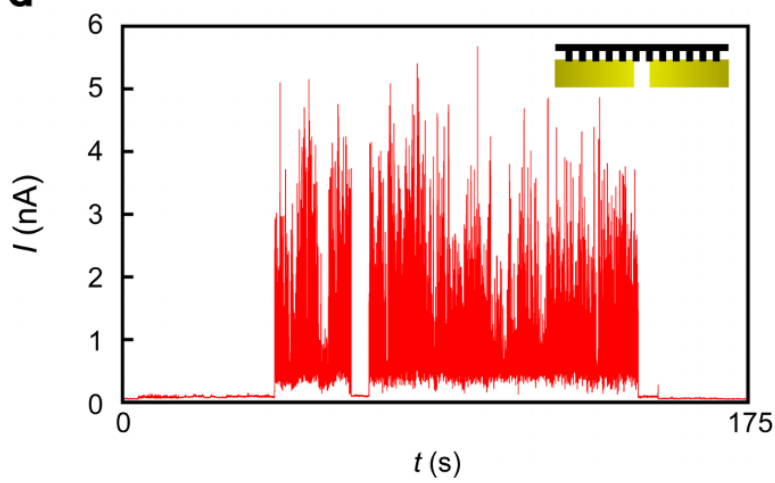

Figure $4 \mid$ DNA length dependence of molecular trapping duration. a, $I$ - $t$ curve acquired in an aqueous solution of $22 \mathrm{mer}$ nucleotides. b, A magnified view of $I$-spikes in (a). The spike width $t_{\mathrm{d}}$ denotes the trapping duration of oligonucleotides in the electrode gap. c, Trapping duration variations of nucleotides with various lengths. The distribution shift monotonically to longer $t_{\mathrm{d}}$ regime with the length of DNA. Solid lines are Gaussian fit. $\mathrm{d}$, Anomalous current signals with abnormally high $I_{\mathrm{p}}$ and long $t_{\mathrm{d}}$, which presumably reflects the longitudinal electron transport through the $22 \mathrm{mer}$ DNA bridge modelled in the inset.

way, the in-plane nanopore detector can potentially be exploited for identifying nucleobases in oligonucleotides.

As long-read is of a major concern in DNA sequencer, we further attempted electrical identification of longer oligonucleotides with 22 -base sequence. The acquired $I-t$ traces $\left(1 \mu \mathrm{M}\right.$ in Milli-Q, $V_{\mathrm{b}}=$ $0.75 \mathrm{~V}$ ) showed current spikes (Fig. $4 \mathrm{a}$ and $4 \mathrm{~b}$ ). These signals had similar amplitudes in a range from $20 \mathrm{pA}$ to $500 \mathrm{pA}$. On the other hand, the dwell time $t_{\mathrm{d}}$ was longer compared to the shorter oligonucleotide counterparts as manifested in Fig. 4c.

\section{Discussion}

The monotonic shift of $t_{\mathrm{d}}$ distributions with increasing the length of DNA oligomers is ascribed a priori to a difference in time necessary for a DNA to flow through an electrode gaps. When this is the case, $t_{\mathrm{d}}$ of $n$-base oligonucleotides should scale as $t_{\mathrm{d}}=t_{\mathrm{m}} \times n$, where $t_{\mathrm{m}}$ is the trapping duration of the monomer. On the other hand, the characteristic $t_{\mathrm{d}}$ estimated by Gaussian fitting is $13 \mathrm{~ms}, 27 \mathrm{~ms}$, and $91 \mathrm{~ms}$ for monomer, 6-mer, and 22-mer nucleotides, respectively. This implies that in most of the cases only about $30 \%$ of the entire length of the oligonucleotides could pass through the electrode gap. Furthermore, peculiar $I$-spikes with extraordinarily long $t_{\mathrm{d}}$ (>100 s) and high $I_{\mathrm{p}}(>5 \mathrm{nA})$ were found for 22-mer nucleotides (Fig. 4d). Similar high-current signals were observed in recent tunnelling current measurements of $\lambda$-DNA. ${ }^{16}$ The anomalous feature most likely arises from longitudinal electron transport through a long DNA connected to two Au electrodes (Fig. 4d, inset). These issues can be resolved technically by introducing the electrophoretic method and making the pore size smaller by reducing the thickness of $\mathrm{Au}$ junction to confine and regulate a DNA flow through the electrode gap. The electrode-embedded in-plane nanopore singlemolecule detector offers the prospect of label-free electrical DNA sequencing.

\section{Methods}

Fabrication of $\mathrm{SiO}_{2} / \mathrm{Au} / \mathrm{SiO}_{2}$ junctions. Micro-electrodes were formed on a $300 \mathrm{~nm}$ thick $\mathrm{SiO}_{2}$ insulating layer on a phosphor-doped $\mathrm{Si}$ substrate using a photolithography method with subsequent deposition of $\mathrm{Cr} / \mathrm{Au}(1 \mathrm{~nm} / 30 \mathrm{~nm})$ by the radio-frequency (RF) magnetron sputtering and lift-off processes. After that, a nano-scale junction and micro-pillar array were patterned using an electron-beam lithography technique. Then, $\mathrm{Au} / \mathrm{SiO}_{2}(15 \mathrm{~nm} / 150 \mathrm{~nm})$ layer was deposited by the RF sputtering, followed by lift-off to form a $\mathrm{Au} / \mathrm{SiO}_{2}$ junction with a bow-tie geometry and square-shaped pillars. Finally, the sample was exposed to isotropic reactive ion etching using $\mathrm{CF}_{4}$ and $\mathrm{O}_{2}$ mixture gas to form a $\mathrm{SiO}_{2}(75 \mathrm{~nm}) / \mathrm{Au}(15 \mathrm{~nm}) / \mathrm{SiO}_{2}(75 \mathrm{~nm})$ junction.

Electromigration breaking. Bias sweep was applied to the $\mathrm{SiO}_{2} / \mathrm{Au} / \mathrm{SiO}_{2}$ junction under a resistance feed-back control using Keithley 6487 picoammeter/voltage source in a vacuum better than $10^{-5}$ Torr. Specifically, a dc voltage $V_{\mathrm{b}}$ imposed to the multilayered junction was increased linearly from $0.2 \mathrm{~V}$ at a rate of $400 \mathrm{mV} / \mathrm{s}$. When $V_{\mathrm{b}}$ becomes higher than $1 \mathrm{~V}$, this rate was decrease to $100 \mathrm{mV} / \mathrm{s}$ to prevent overcurrent junction breakdown. The junction resistance $R$ tended to increase at high-field by concentrated Joule heating and associated thermally-activated electromigration at its narrowest constriction region. After the resistance increased by $0.4 \%$ from that at $V_{\mathrm{b}}=0.2 \mathrm{~V}, V_{\mathrm{b}}$ was quickly lowered to $0.2 \mathrm{~V}$. The junction could be thinned gradually to atomic scale by repeating these automated processes until $R$ became larger than $1000 \Omega$. The active electromigration-induced thinning process was then halted and the metastable atom-sized contact was broken by thermal fluctuations at a low constant bias voltage of $V_{\mathrm{b}}=0.1 \mathrm{~V}$. At this stage, we observed the conductance $G$ to drop in a stepwise manner, indicative of thermally-activated breakdown of Au atom-sized contact (Fig. 1b, see also Supplementary Fig. S1 and S2). Right before the eventual breakdown, a long plateau was often observed at $G \sim 1 G_{0}$ $\left(G_{0}=2 e^{2} / h \sim 77.5 \mu \mathrm{S}\right.$ is the conductance quantum), which signifies that Au junction has been narrowed to one atom size wherein one fully transparent channel exists for electron transmission. ${ }^{30}$ What followed was structure relaxation after self-breaking of Au single-atom contacts and accompanied formation of a sub-nanometer electrode gap. Only when an electrode gap was formed in this way did we detected the characteristic current spikes in molecular solution; $I$ - $t$ curves were totally featureless when overcurrent breakdown occurred during the electrical breaking processes that results in large electrode gaps (see Supplementary Fig. S6).

PDMS channel. An SU-8 mold with a micro-channel pattern was formed on a $\mathrm{SiO}_{2} /$ Si wafer by a photolithography procedure. Subsequently, the PDMS (Sylgard 184) was cured on the mold in an oven at 70 degrees Celsius. The PDMS replica was then cut 
and peeled off from the mold. The thus prepared PDMS channel was bonded on the sample substrate onto which a $\mathrm{SiO}_{2} / \mathrm{Au} / \mathrm{SiO}_{2}$ junction and micro-pillars were formed by pretreating the both surfaces with oxygen plasma and UV light for better adhesion and enhanced hydrophilic properties.

Tunnelling current measurements. After forming an electrode-embedded nanopore structure by the electromigration/self breaking technique in a vacuum, Argon gas was admitted to the sample chamber and a dilute solution of a target molecule current was injected into the PDMS channel. The nucleotide molecules were synthesized by FASMAC Co., Ltd. Er-encapsulated $\mathrm{C}_{82}$ molecules were synthesized using the scheme reported elsewhere. ${ }^{21}$ Chrolobenzene was purchased from Sigma-Aldrich Co., Ltd. Electrical current flowing through the two Au nanoelectrodes was then recorded at a sampling rate of $10 \mathrm{kHz}$ with a home-built current preamplifier and a National Instruments PXI-5922 digitizer under a constant dc bias voltage $V_{\mathrm{b}}$. The amplifier is a logarithmic current-to-voltage converter with a linear transfer characteristics below $1 \mathrm{nA}$ and a bandwidth of $100 \mathrm{kHz}$, whose basic structure is composed of a sensing resistor and series of four diodes. ${ }^{31}$ The current measurements were carried out not more than consecutive 2 hours to avoid possible contamination effects.

1. Branton, D. et al. The potential and challenges of nanopore sequencing. Nat Biotech. 26, 1146-1153 (2008).

2. Zwolak, M. \& Di Ventra, M. Colloquim: Physical approaches to DNA sequencing and detection. Rev. Mod. Phys. 80, 141-165 (2008).

3. Dekker, C. Solid-state nanopores. Nat. Nanotechnol. 2, 209-215 (2007).

4. Hall, A. R., Scott, A., Rotem, D., Mehta, K. K., Bayley, H. \& Dekker, C. Hybrid pore formation by directed insertion of $\alpha$-haemolysin into solid-state nanopores. Nat. Nanotechnol. 5, 874-877 (2010).

5. Clarke, J., Wu, H.-C., Jayasinghe, L., Patel, A., Reid, S. \& Bayley, H. Continuous base identification for single-molecule nanopore DNA sequencing. Nat. Nanotechnol. 4, 265-270 (2009).

6. Derrington, I. M. et al. Nanopore DNA sequencing with MspA. Proc. Nat. Acad. Sci. 107, 16060-16065 (2010).

7. Kasianowicz, J. J., Brandin, E., Branton, D. \& Deamer, D. W. Characterization of individual polynucleotide molecules using a membrane channel. Proc. Nat. Acad. Sci. 93, 13770-13773 (1996).

8. Garaj, S., Hubbard, W., Reina, A., Kong, J., Branton, D. \& Golovchenko, J. A. Graphene as a subnanometre trans-electrode membrane. Nature 467, 190-193 (2010)

9. Keyser, U. F. et al. Direct force measurements on DNA in a solid-state nanopore. Nat. Phys. 2, 473-477 (2006).

10. McNally, B., Singer, A., Yu, Z., Sun, Y., Weng, Z. \& Meller, A. Optical recognition of converted DNA nucleotides for single-molecule sequencing using nanopore arrays. Nano Lett. 10, 2237-2244 (2010).

11. Liang, X. \& Chou, S. Y. Nanogap detector inside nanofluidic channel for real-time lavel-free DNA analysis. Nano Lett. 8, 1472-1476 (2008).

12. Tsutsui, M., Taniguchi, M., Yokota, K. \& Kawai, T. Identifying single nucleotides by tunnelling current. Nat. Nanotechnol. 5, 286-290 (2010).

13. Huang, S. et al. Identifying single bases in a DNA oligomer with electron tunnelling. Nat. Nanotechnol. 5, 868-873 (2010).

14. Lagerqvist, J., Zwolak, M. \& Di Ventra, M. Fast DNA sequencing via transverse electronic transport. Nano Lett. 6, 779-782 (2006).

15. Taniguchi, M., Tsutsui, M., Yokota, K. \& Kawai, T. Fabrication of the gating nanopore device. Appl. Phys. Lett. 95, 123701 (2009).

16. Ivanov, A. P. et al. DNA tunneling detector embedded in a nanopore. Nano Lett. 11, 279-285 (2011)

17. O’Neill, K., Osorio, E. A. \& van der Zant, H. S. J. Self-breaking in planar few-atom Au Constrictions for nanometer-spaced electrodes. Appl. Phys. Lett. 90, 133109 (2007)
18. Tsutsui, M., Taniguchi, M. \& Kawai, T. Fabrication of $0.5 \mathrm{~nm}$ electrode gaps using self-breaking technique. Appl. Phys. Lett. 93, 163115 (2008).

19. Kanaya, K. \& Okayama, S. Penetration and energy-loss theory of electrons in solid targets. J. Phys. D: Appl. Phys. 5, 43 (1972).

20. Trouwborst, M. L., van der Molen, S. J. \& van Wees, B. J. The role of Joule heating in the formation of nanogaps by electromigration. J. Appl. Phys. 99, 114316 (2006).

21. Madams, C. J., Morgan, D. V. \& Howes, M. J. Migration of gold atoms through thin silicon oxide films. J. Appl. Phys. 45, 5088-5090 (1974).

22. Taychatanapat, T., Bolotin, K. I., Kuemmeth, F. \& Ralph, D. C. Imaging electromigration during the formation of break junctions. Nano Lett. 7, 652-656 (2007).

23. Hirvonen, J. K., Weisenberger, W. H., Westmoreland, J. E. \& Meussner, R. A Backscattering investigation of low-tempearture migration of chromium through gold films. Appl. Phys. Lett. 21, 37-39 (1972).

24. Shinohara, H. Endohedral metallofullerenes. Rep. Prog. Phys. 63, 843-892 (2000).

25. Chavy, C., Joachim, C. \& Altibelli, A. Interpretation of STM images: $C_{60}$ on the gold (110) surface. Chem. Phys. Lett. 214, 567 (1993).

26. Park, H., Park, J., Lim, A. K. L., Anderson, E. H., Alivisatos, A. P. \& McEuen, P. L. Nano-mechanical oscillations in a single- $\mathrm{C}_{60}$ transistor. Nature $407,57-60$ (2000).

27. Xu, B. \& Tao, N. J. Measurement of single-molecule resistance by repeated formation of molecular junctions. Science 301, 1221 (2003).

28. Martin, C. A., Ding, D., Sørensen, J. K., Bjørnholm, T., van Ruitenbeek, J. M. \& van der Zant, H. S. J. Fullerene-based anchoring groups for molecular electronics. J. Am. Chem. Soc. 130, 13198-13199 (2008).

29. Chang, S. et al. Electronic signatures of all four DNA nucleosides in a tunneling gap. Nano Lett. 10, 1070-1075 (2010)

30. Agrait, N., Yeyati, A. L. \& van Ruitenbeek, J. M. Quantum properties of atomicsized conductors. Phys. Rep. 377, 81-279 (2003).

31. Dürig, U., Novotny, L. \& Michel, B. Logarithmic current-to-voltage converter for local probe microscopy. Rev. Sci. Instrum. 68, 3814-3816 (1997).

\section{Acknowledgements}

This work was supported by the Japan Society for the Promotion of Science (JSPS) through its "Funding Program for World-Leading Innovative R\&D on Science and Technology".

\section{Author contributions}

M. Tsutsui, M. Taniguchi, and T. K. planned and designed experiments. M. Tsutsui and R. S. fabricated in-plane nanopore devices. M. Tsutsui and M. Taniguchi exhibited single-molecule conductance measurements. Y. I. and T. O. synthesized metal-encapsulated fullerenes. M. Tsutsui and M. Taniguchi performed data analyses. M. Tsutsui, M. Taniguchi, and T. K. co-wrote paper.

\section{Additional information}

Supplementary Information accompanies this paper at http://www.nature.com/ scientificreports

Competing financial interests: The authors declare no competing financial interest. License: This work is licensed under a Creative Commons Attribution-NonCommercial-ShareAlike 3.0 Unported License. To view a copy of this license, visit http://creativecommons.org/licenses/by-nc-sa/3.0/

How to cite this article: Tsutsui, M. et al. Single-molecule sensing electrode embedded in-plane nanopore. Sci. Rep. 1, 46; DOI:10.1038/srep00046 (2011). 